\title{
Evaluation of Bond Strength \& Enamel Alterations with the Contemporary Light Cure Orthodontic Adhesives: A Scanning Electron Microscope Study
}

\section{Kamboj $\mathbf{A}^{1}$, Chopra $S^{2}$, Mitra $\mathbf{R}^{2}$, Jayan $\mathrm{B}^{3}$, Datana $\mathrm{S}^{1}$ and Agarwal SS $1 *$}

${ }^{1}$ Department of Orthodontics \& Dentofacial Orthopedics, Armed forces medical college, India

${ }^{2}$ Department of Orthodontics \& Dentofacial Orthopedics, Army Dental Centre R\&R, India

${ }^{3}$ Department of Orthodontics \& Dentofacial Orthopedics, Air Force Institute of Dental Sciences, India

*Corresponding author: SS Agarwal, Asst Professor, Department of Orthodontics \& Dentofacial Orthopedics, Armed forces medical college, Pune, India, Tel: 007086104044; Email: docshivagarwal@rediffmail.com

\section{Abstract}

Introduction: The introduction of acid etch technique in orthodontics has partially eliminated the banding technique due to factors like conservation of band space, prevention of decalcification beneath the loose bands and improved aesthetics and decreased cost. Two paste system, no mix adhesive, light activated direct bonding material and adhesive pre-coated brackets are present modifications of adhesive formulations.

Materials and Methods: The sample of this in-vitro study consisted of ninety caries free, intact extracted mandibular first molar teeth. The roots of the teeth were embedded in the centre of a cold cure acrylic. A molar tube of 0.018" Roth prescription with micro etched base was bonded to each molar with three different bonding agents. All the molar tubes were subsequently tested for evaluation of shear bond strength with a universal testing machine. Scanning Electron Microscope (SEM) was used to evaluate the effect of various light cure adhesives on the enamel morphology.

Results: Transbond-XT exhibited the highest bond strength (22.6 MPa) closely followed by Transbond Plus SEP (19.6 $\mathrm{MPa})$ and GC Fuji Ortho LC (13.89 MPa).

Conclusions: The adequate bond strength of glass ionomers, less deleterious effects on the enamel and sustained fluoride release make it a good option for routine use in orthodontics.

Keywords: Transbond-XT; Transbond Plus SEP; GC Fuji Ortho LC; Shear bond strength 
Abbreviations: SEM: Scanning Electron Microscope; SEP: Self-Etching Primer; ANOVA: Analysis of Variance; MPa: Megapascals; SEM: Scanning Electron Microscope; ESEM: Environmental Scanning Electron Microscope.

\section{Background}

The acid etch technique was first suggested by Michael G Buonocore in 1955 [1,2]. George V Newman in 1965 [3] experimentally and clinically bonded plastic orthodontic attachments directly to enamel using acid etch technique with epoxy resins and curing agents and the process was known as bonding. Its introduction in orthodontics has eliminated the banding technique due to factors like conservation of band space, prevention of decalcification beneath the loose bands, improved aesthetics and decreased cost. The two paste system, no mix adhesive, light activated direct bonding material and adhesive precoated brackets are the present modifications of adhesive formulations.

Today's advanced adhesives coupled with high performance, light force wires have allowed clinicians to greatly expand the use of direct bond tubes on the molars. These reduce inventory requirements, are often more comfortable and hygienic for the patient and bond and debond quickly and easily. Recent modifications in molar tube fabrication have led to the production of bonding bases contoured to the buccal enamel, which facilitate placement and are likely to enhance adhesion with a bonding agent and promote better bond reliability. A review of the literature reveals that the use of molar tubes in conjunction with newer light cure bonding agent has not been thoroughly evaluated. The objective of this invitro study was to determine the shear bond strength of orthodontic molar tubes bonded with three different light cure bonding agents and to evaluate and compare the changes in the surface morphology of debonded enamel surface under the SEM.

\section{Materials and Methods}

The sample of this in-vitro study consisted of ninety caries free, intact extracted mandibular first molar teeth. These teeth were cleaned of residual soft tissue debris under running water with a tooth brush and then decontaminated in $10 \%$ formalin for one week. The teeth were then stored in distilled water. The roots of the teeth were embedded in the centre of a cold cure acrylic (DPIRR Products Ltd) block measuring $12 \mathrm{~mm} \mathrm{X} 12 \mathrm{~mm} \mathrm{X} 25$ $\mathrm{mm}$, with the crowns of the teeth exposed in such a way that the long axis of the tooth was vertical with the buccal surface exposed for the bonding procedure. All the teeth were randomly assigned to three groups with thirty teeth in each group. A colour code was used for each group (Table 1).

\begin{tabular}{|c|c|c|}
\hline Group & Name of the Group & Colour Code \\
\hline Group-A (1-30) & Transbond-XT & Yellow \\
\hline Group-B (31-60) & Transbond Plus SEP & Red \\
\hline Group-C (61-90) & G.C. Fuji Ortho LC & Grey \\
\hline
\end{tabular}

Table 1: Colour coding of groups

The buccal surface of each mounted tooth was cleaned with fluoride free pumice slurry in an aqueous base with a rubber cup on contra-angle hand piece with slow speed for 5 seconds. They were then washed for 10 seconds in distilled water with a three way air syringe and dried for 10 seconds with oil free and moisture free gentle air spray. A molar tube of 0.018 " Roth prescription with micro etched base (Navy bondable tubes sourced from Libral Traders Ltd) Figure 1 was bonded to each molar for each bonding agent and the bonding procedure recommended by the manufacturer was followed. The molar tubes had a buccal groove indicator to facilitate placement and a contoured base to aid adaptation to the buccal enamel surface.

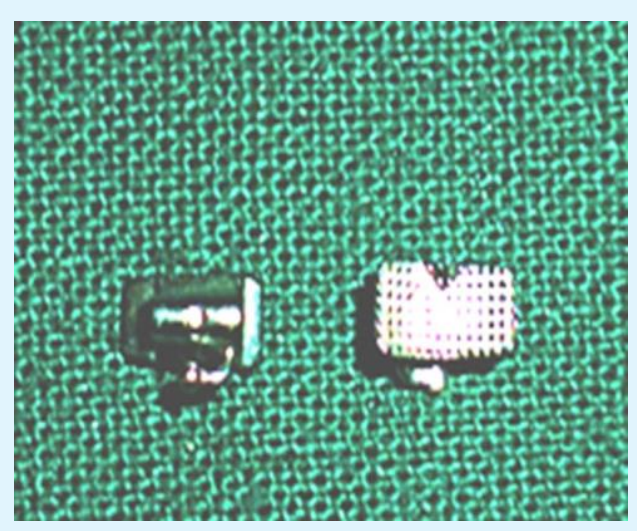

Figure 1: 0.018" Preadjusted Edgewise Roth Molar Tubes

\section{Bonding Procedure for Group A}

Acid etching was done with $37 \%$ orthophosphoric acid (Scotchbond, 3M Unitek) for 30 seconds (Figure 2). Thereafter the tooth surface was rinsed with water for 30 seconds and dried with moisture free air to get a frosty white appearance of tooth surface. Transbond XT primer was coated on the etched tooth surface with an applicator tip. The primer coating was thinned with a gentle air burst directed gingivo-occlusally for 1-2 seconds. Transbond XT adhesive paste (3M Unitek) was applied to 
the bonding base of the molar tube. The tube was placed on the tooth surface, excess material was removed with an explorer and the adhesive was cured with a LED light source.

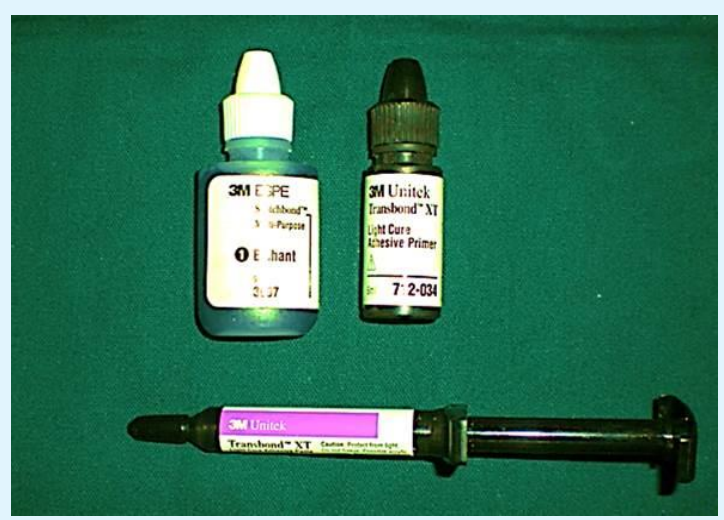

Figure 2: Transbond TM XT (Primer and Adhesive) \& Scotchbond TM (Etchant).

\section{Bonding Procedure for Group B}

The single use package of Transbond Plus Self-Etching Primer (SEP) Figure 3 was used for etching and priming. This primer consisted of three compartments. The first compartment contained methacrylated phosphoric acid esters, photosensitizers and stabilizers. The second compartment contained water and soluble fluoride. The third compartment contained an applicator microbrush. Squeezing and folding the first compartment over to the second activated the system. The mixed component in the second compartment was then ejected into the third compartment to wet the applicator tip. The tip was rubbed on the entire buccal enamel surface for a minimum of 3-5 seconds. An oil and moisture free air source was then used to deliver a gentle air burst for 1-2 seconds to dry the primer into a thin film on each tooth.

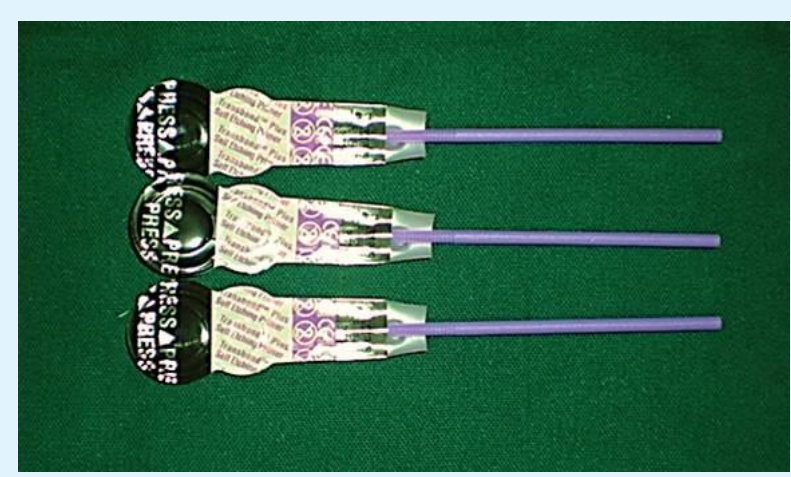

Figure 3: Transbond TM Plus SEP (Etchant And Primer Combination).
Then light cure composite adhesive paste (TransbondXT) was applied to the bonding base of the molar tube, the tube was placed on the tooth surface lightly with the help of a bracket holding forceps, final position of the tube was adjusted and it was pressed firmly. Excess material was removed with an explorer and the adhesive was cured with the LED light source.

\section{Bonding Procedure for Group C}

G.C. Fuji conditioner was applied for twenty seconds to the bonding surface of the tooth with the help of an applicator tip. The tooth was then rinsed thoroughly with water. However, after rinsing, the bonding surface was not thoroughly dried and was kept moist during the bonding procedure, by rehydrating with moistened cotton roll which was applied with a single stroke occlusogingivally. After this, the cement was mixed in the powder and liquid ratio of $3.6 \mathrm{~g} / 1.0 \mathrm{~g}$ i.e. one level of large scoop of powder to two drops of liquid in a mixing pad (Figure 4). The powder was divided into two equal parts. The first half was mixed with the entire liquid for approximately 10 seconds and then the remaining half was also added. Once optimal mix was obtained, the bonding surface of the tube was completely coated with mixed cement and then the tube was placed firmly against the enamel surface and cured with the LED light source. Curing was done for 10 seconds on each side i.e. mesial and distal with the light tip kept at a distance of $2 \mathrm{~mm}$ from the molar tube.

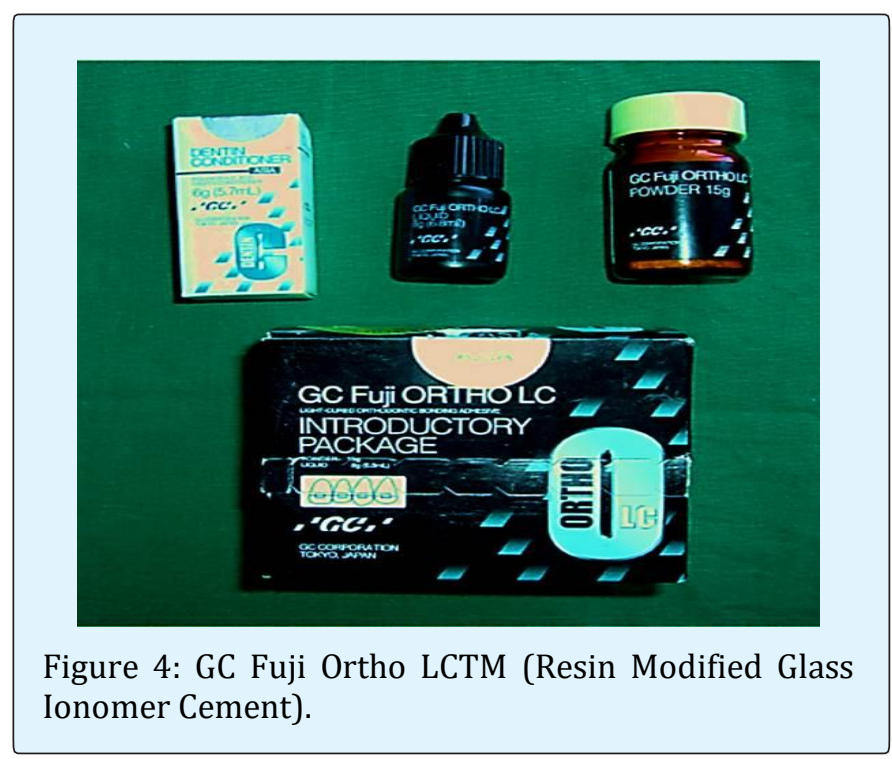

The LED light source used for all three groups was EMorlit, Taiwan with an intensity of $900 \mathrm{~mW} / \mathrm{cm} 2$ as measured with lux meter. On completion of the bonding 
procedure Figures 5(a-c) all specimens were kept in distilled water Figure 6 for 24 hours at room temperature

after which debonding of the molar tubes was carried out.

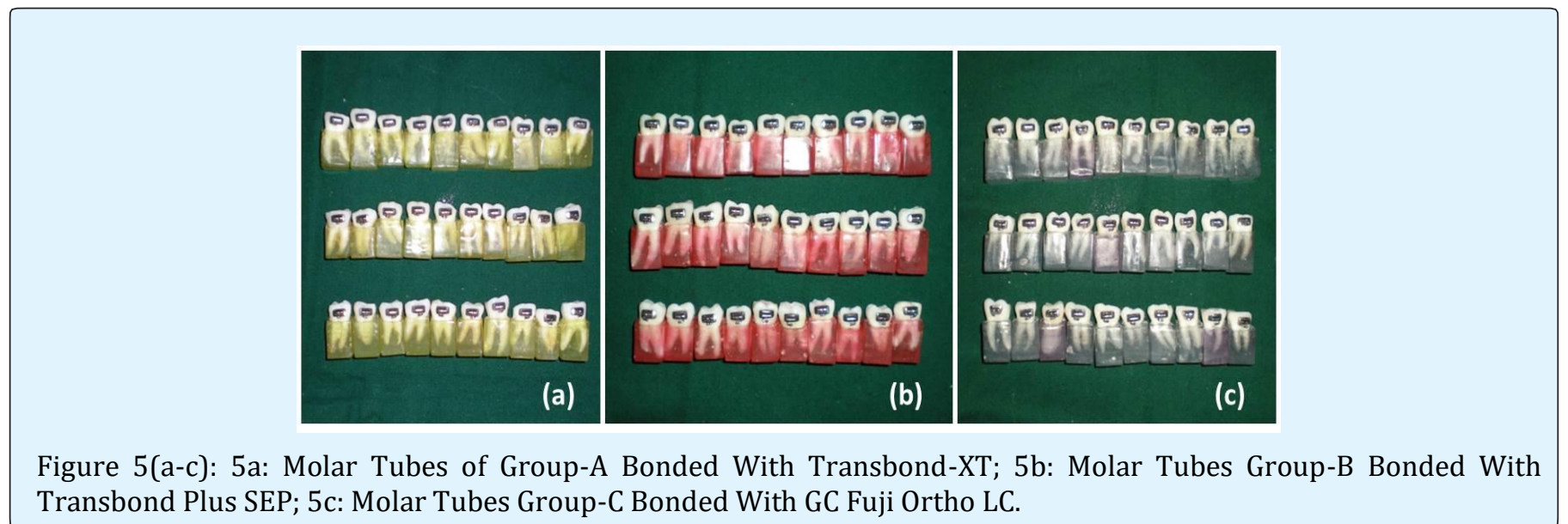

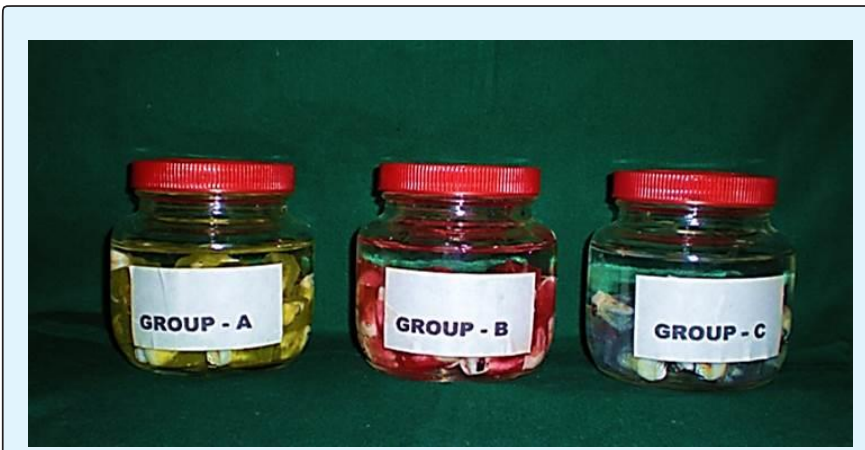

Figure 6: All Specimens Stored In Distilled Water.

\section{Preparation of Bonded Tooth for Debonding Test for Shear Bond Strength}

All the molar tubes were subsequently tested for evaluation of shear bond strength with a universal testing machine (Model H25KS, Germany), Figure 7. First, measurement of the bondable surface area of the molar tube was done using a digital vernier calliper (Mitutoyo Miyazaki, Japan), Figure 8 that was found to be $18 \mathrm{~mm}^{2}$. The universal testing machine was connected to a dedicated computer which was used to switch on the machine. The necessary test method from the "Test Zone" programme depending upon the need was selected (shear bond strength in the present study). The test parameters data namely load $(5000 \mathrm{~N})$, range (5.30kgf), gauge length $(0.01 \mathrm{~mm})$, speed of test $(1 \mathrm{~mm} / \mathrm{min})$ and the approach speed $(0.50 \mathrm{~mm} / \mathrm{min})$ was entered into the computer.

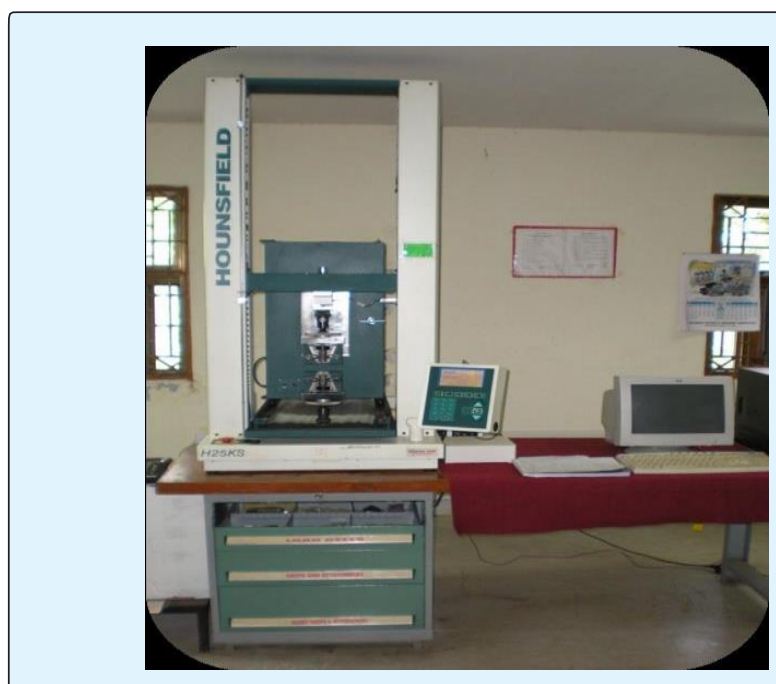

Figure 7: Universal Testing Machine.

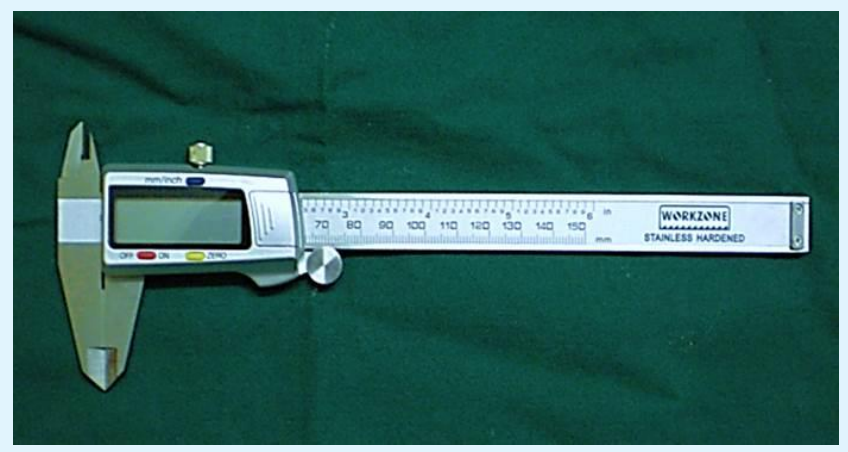

Figure 8: Digital Vernier Caliper. 
The custom made knife-edge metallic jig Figure 9 was positioned in the upper jaw of the machine using a heavy grip in such a way that the edge of the jig made contact with the bonded specimen between the molar tube base and the buccal surface of the teeth. The specimen to be tested was loaded in the lower jaw of the machine using a heavy grip Figures 10(a-c). Each sample was stressed in the occulsogingival direction at a crosshead speed of $1 \mathrm{~mm}$ per minute. The load was increased automatically by the machine till the break point was reached. This was displayed graphically on the monitor of the computer. The bond strength was determined in the shear mode until debonding took place. The values of failure loads were recorded in megapascals (MPa).

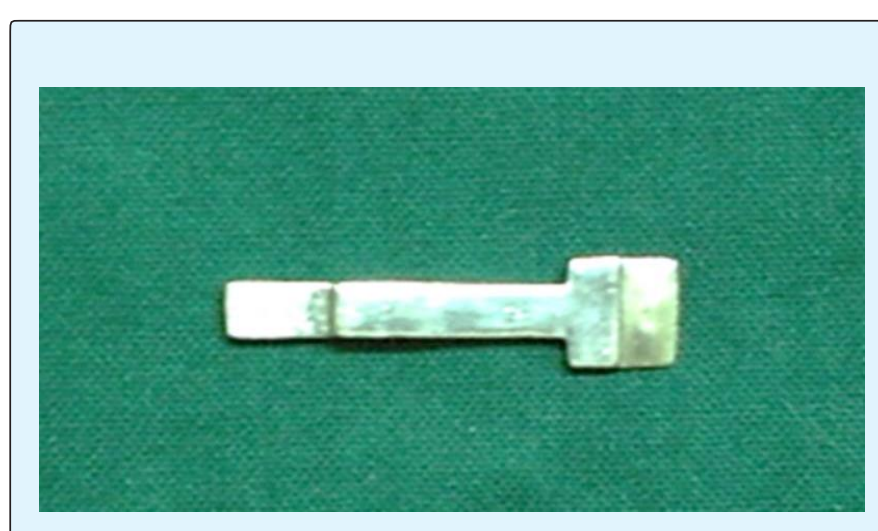

Figure 9: Metal Jig.

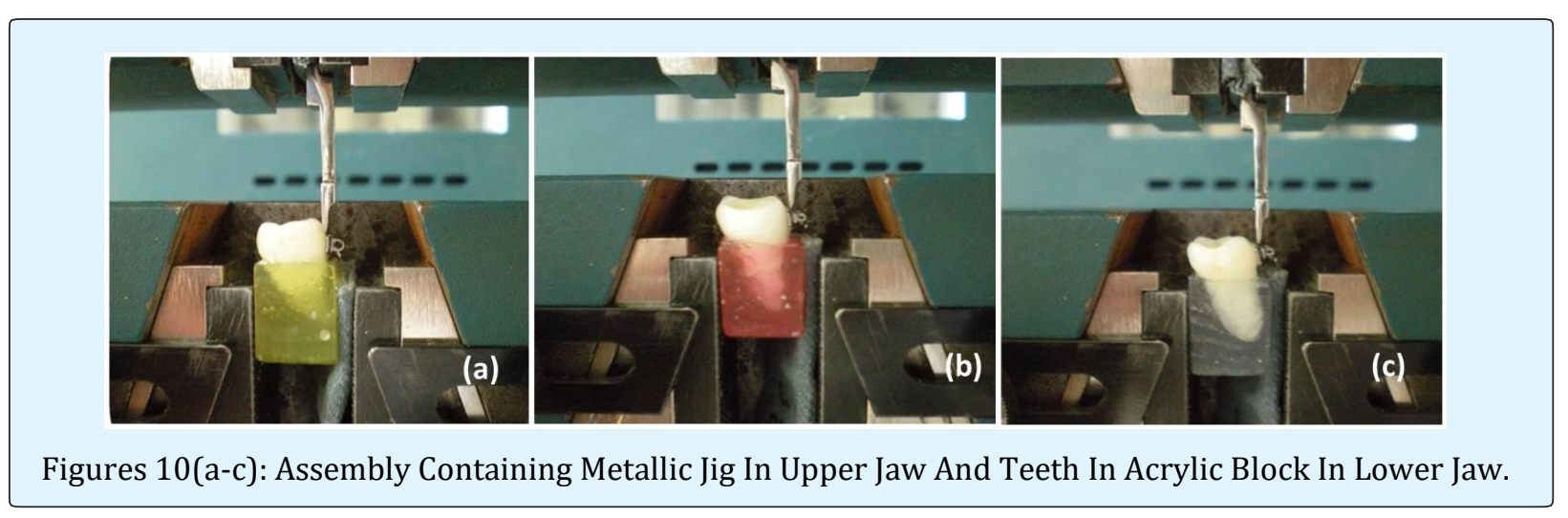

\section{Scanning Electron Microscope (SEM) Analysis}

SEM was used to evaluate the effect of various light cure adhesives on the enamel morphology. Three samples from each of the three Groups were selected randomly to visualise the resin penetration into the enamel surface using scanning electron microscope. Quanta- 200 (Fei, Netherlands), (Figure 11).

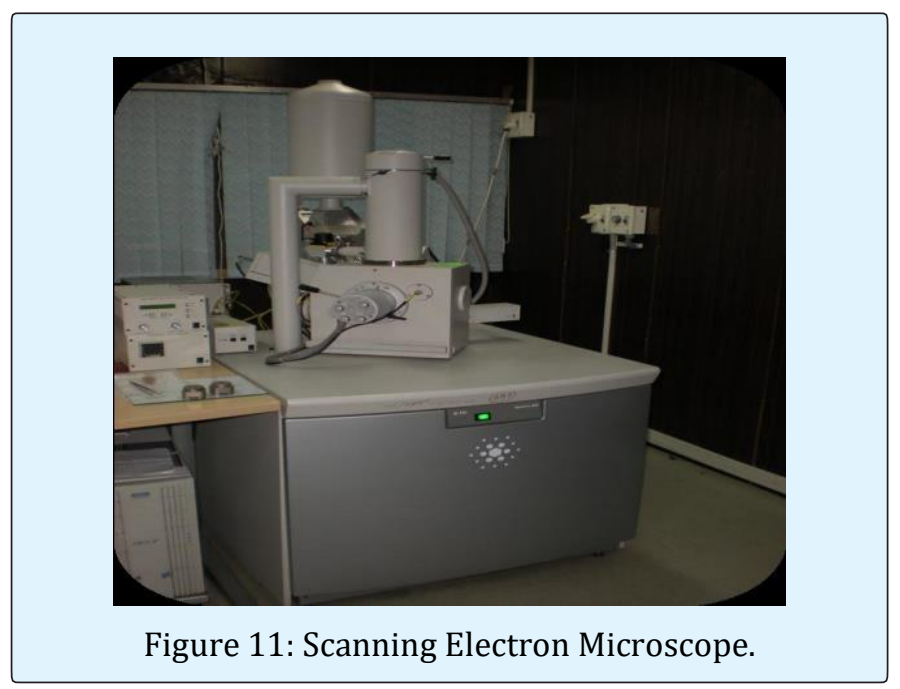

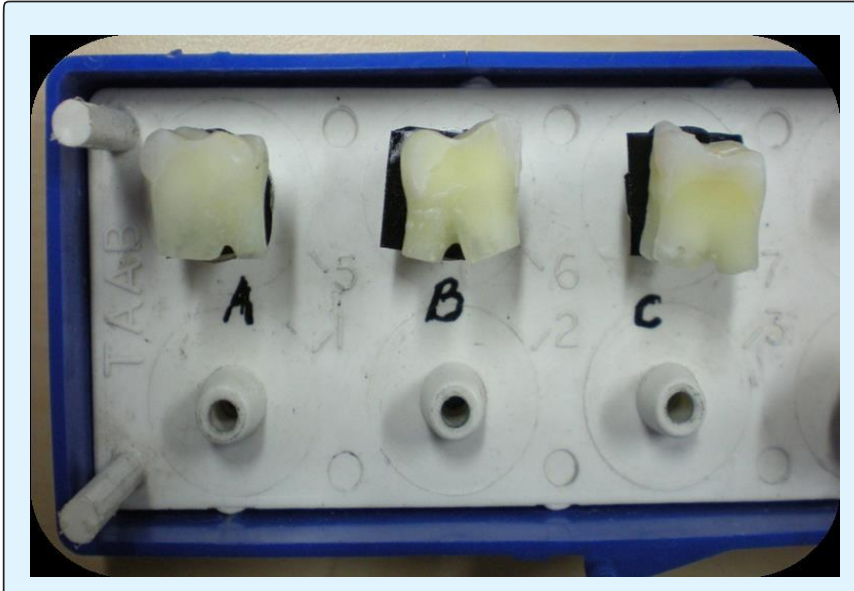

Figure 12: Specimens Loaded On Aluminium Stubs.

These crowns post debonding were transversely sectioned from the roots embedded in the acrylic block with the help of a carborundum disc of $15 \mathrm{~mm}$ diameter (SS White Ltd.) loaded on the straight hand piece of a micromotor (Confident India Ltd.) by a single operator followed by sectioning mesiodistally in a vertical direction from the occlusal to cervical, using the same 
carborundum disc, hand piece and micromotor. The sectioning was carried out under a constant stream of distilled water spray using a $10 \mathrm{ml}$ hypodermic syringe (Dispovan). Now, the specimens were loaded on aluminium stubs using double sided graphite adhesive tape and marking for samples A, B \& C was done with a coloured marker (Figure 12).
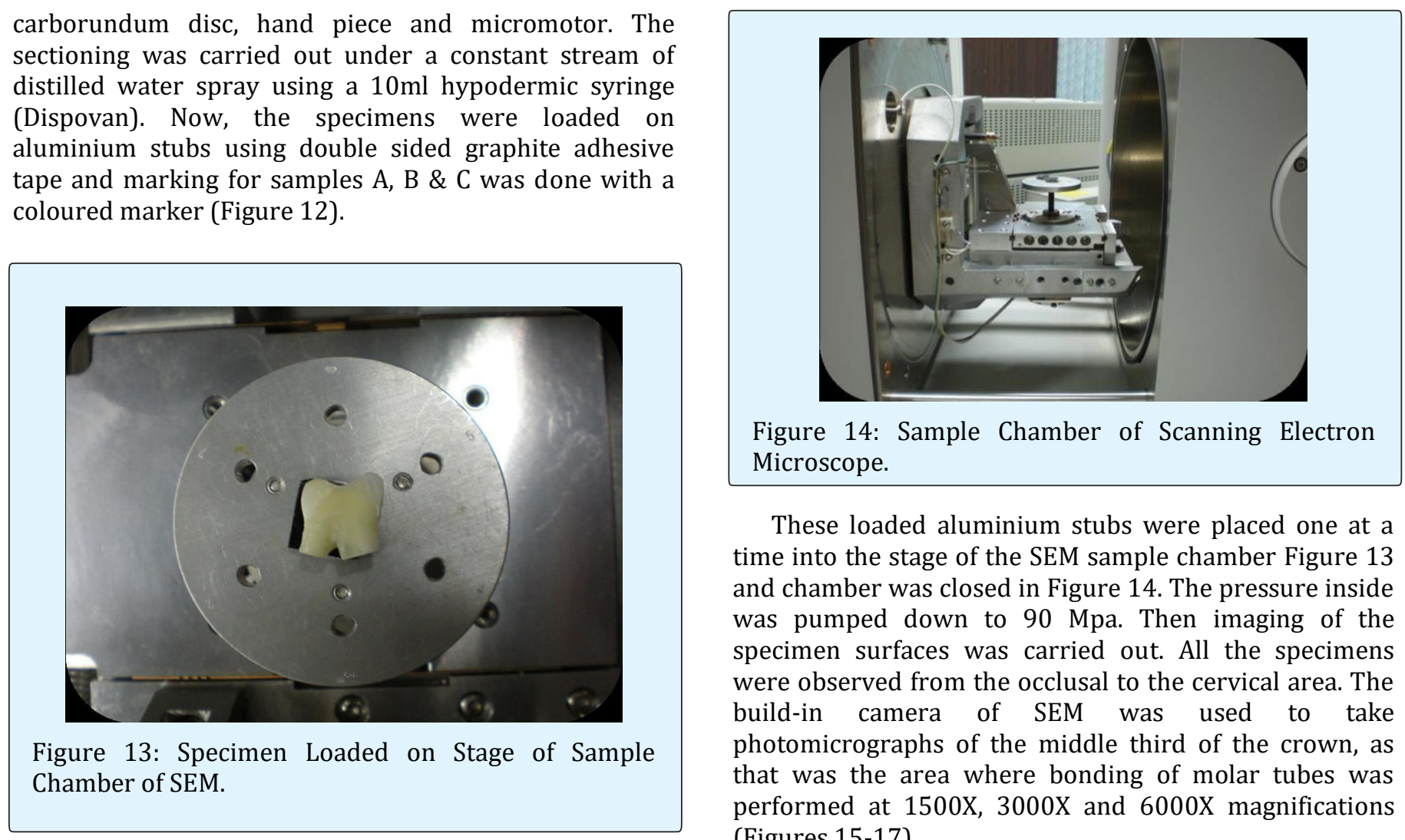

These loaded aluminium stubs were placed one at a time into the stage of the SEM sample chamber Figure 13 and chamber was closed in Figure 14. The pressure inside was pumped down to $90 \mathrm{Mpa}$. Then imaging of the specimen surfaces was carried out. All the specimens were observed from the occlusal to the cervical area. The build-in camera of SEM was used to take photomicrographs of the middle third of the crown, as that was the area where bonding of molar tubes was performed at 1500X, 3000X and 6000X magnifications (Figures 15-17).

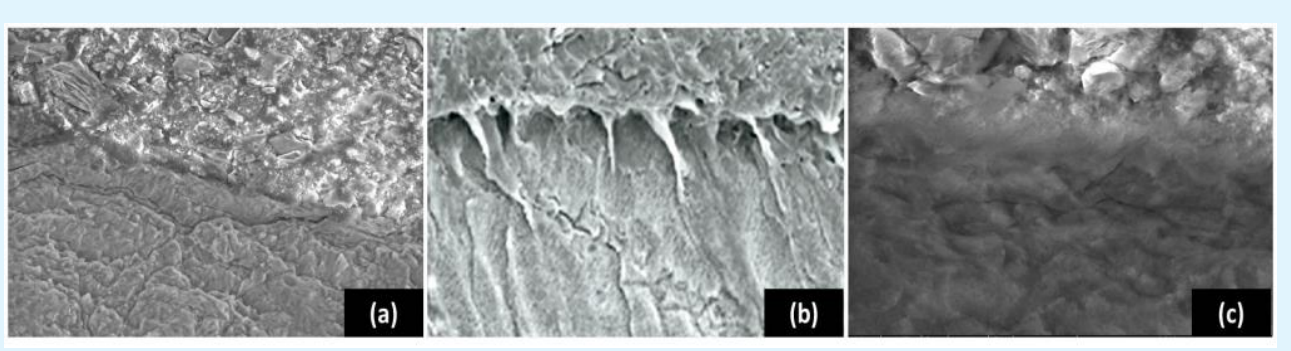

Figure 15(a-c): Group-A (Transbond-XT) 15a: Enamel adhesive interface showing cracks in the enamel surface at 1500X; 15b: Enamel adhesive interface showing resin tags penetrating enamel surface at 3000X; 15c: Enamel adhesive interface showing irregular enamel surface at 6000X.
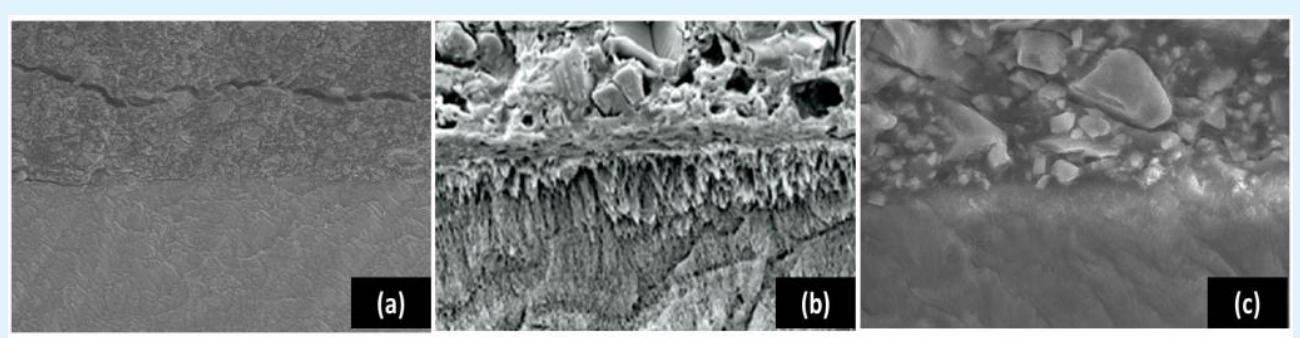

Figure 16(a-c): Group-B (Transbond Plus SEP) 16a: Enamel adhesive interface showing cracks in the adhesive structure at 1500X; 16b: Enamel adhesive interface showing thinner and smaller resin tags at 3000X; 16c: Enamel adhesive interface in high magnification at 6000X. 


\section{Open Access Journal of Dental Sciences}

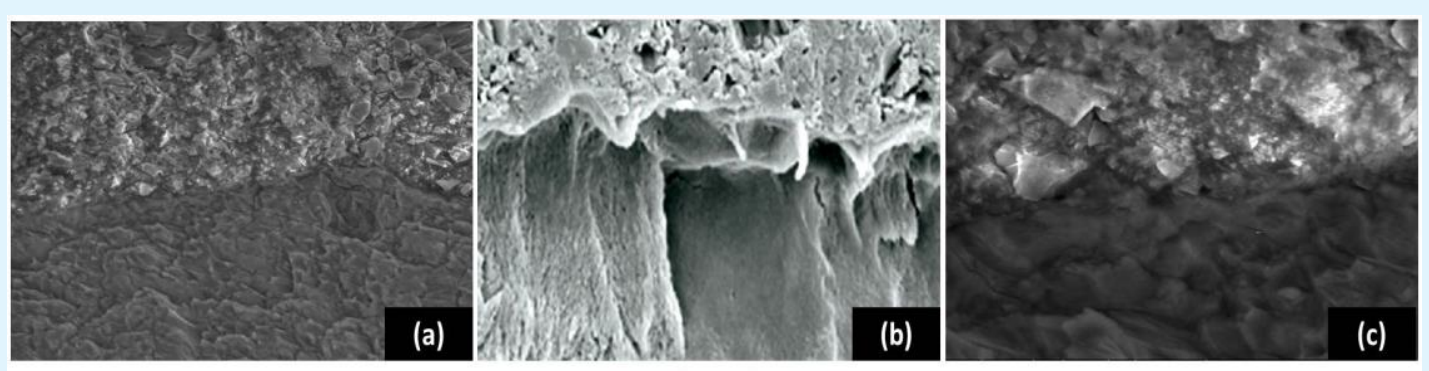

Figure 17: Group-C (G.C. Fuji Ortho LC) 17a: Enamel adhesive interface showing no cracks in the enamel at 1500X; 17b: Enamel adhesive interface showing absence of micro tags 3000X; 17c: Enamel adhesive interface showing large filler particles of the glass ionomer under high magnification at $6000 \mathrm{X}$.

\section{Results}

MINITAB-16 software was used for statistical analysis. Comparison of the mean shear bond strength in MPa with respect to the three bonding materials was done (Graph 1). Transbond-XT exhibited the highest bond strength (22.6 MPa) closely followed by Transbond Plus SEP (19.6 $\mathrm{MPa})$ and GC Fuji Ortho LC (13.89 MPa) at the least. The trend of the mean breaking load of the bonding materials is similar to mean shear bond strength. To evaluate the difference in the mean shear bond strength of the bonding materials, a One Way Analysis of Variance (ANOVA) test was conducted Table 2 that showed that the differences in the mean shear bond strength were statistically very highly significant $(\mathrm{F}=60.15, \mathrm{p}=0.0001)$.The group mean and standard deviations of the three bonding materials were calculated (Table 3). Since the ANOVA test showed significant differences in the mean shear bond strength of the bonding materials, a pair wise comparison for each pair of bonding materials was carried out by using
Turkey's test. The differences of means in each pair were statistically very highly significant. Although mean shear bond strength of Group A and Group B differs only by $2.654 \mathrm{MPa}$, this also is statistically significant.

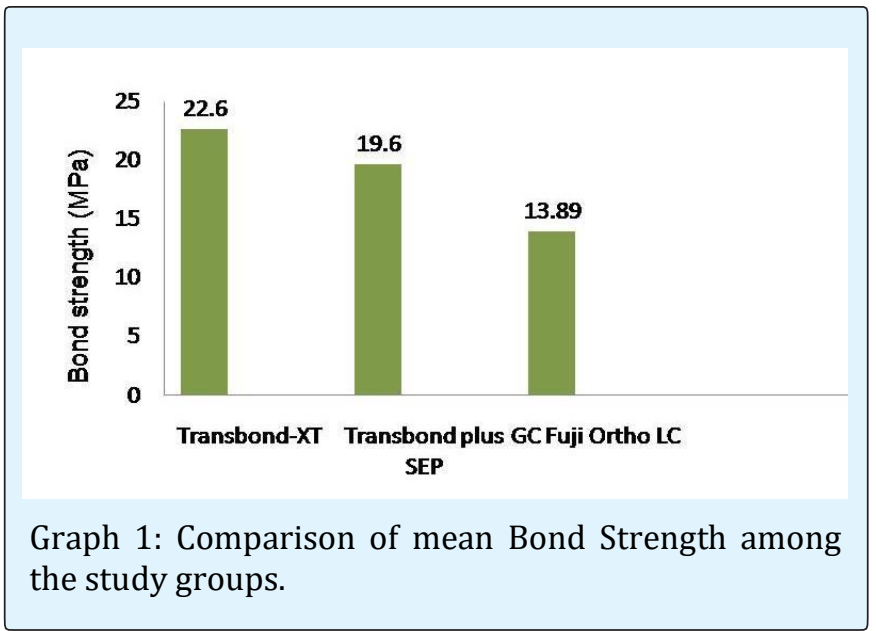

\begin{tabular}{|c|c|c|c|c|c|}
\hline Source & $\begin{array}{c}\text { Degree of } \\
\text { freedom }\end{array}$ & $\begin{array}{c}\text { Sum of } \\
\text { squares }\end{array}$ & $\begin{array}{c}\text { Mean sum of } \\
\text { squares }\end{array}$ & Variance Ratio (f) & $\begin{array}{c}\text { P value or level of } \\
\text { significance (p) }\end{array}$ \\
\hline Groups & 2 & 1198.22 & 599.11 & 60.15 & 0.0001 \\
\hline Error & 87 & 866.55 & 9.96 & - & - \\
\hline Total & 89 & 2064.77 & - & - & - \\
\hline
\end{tabular}

Table 2: One-Way ANOVA: Bond Strength (MPa) Versus Groups.

\begin{tabular}{|c|c|c|c|}
\hline Groups & N & Mean & Standard Deviation \\
\hline Group A & 30 & 22.616 & 3.404 \\
\hline Group B & 30 & 19.962 & 3.421 \\
\hline Group C & 30 & 13.898 & 2.567 \\
\hline
\end{tabular}

Table 3: Comparison of Group Mean and Standard Deviation of Bond Strength. 


\section{Open Access Journal of Dental Sciences}

\section{Scanning Electron Microscope Evaluation of Enamel Adhesive Interface}

Group-A, treated with Transbond-XT, the SEM images showed an interface between enamel and adhesive with many long, thick resin tags that had penetrated into the enamel surface to the length that varied from a few microns to more than 20 microns (Figure 15b). The typical prism structure of the enamel was lost not only on the superficial surface but also in the deeper parts not reached by the resin tags. There were cracks observed on the superficial enamel surface. These findings were consistent in all the three magnifications Figures 15 (a-c). Group-B, treated with the Transbond Plus SEP showed resin tags which were thinner, fewer and shorter than those seen when the teeth were exposed to phosphoric acid (Figure 16b). Cracks were observed in the adhesive structure however there were no cracks visible in the enamel structure (Figure 16a). Group-C, exposed to GC Fuji Ortho LC showed no visible tags (Figure 17b). A distinct border between the enamel and the glass ionomer appeared. The enamel prisms on the surface were almost unaffected (Figure 17a). Under higher magnifications, the large filler particles of the glass ionomer could be seen (Figure 17c). However, some tiny needles less than 1 micrometer projecting into the enamel surface were visible. Whether this belonged to the resin component of the glass ionomer or to the enamel structure could not be assessed with this technique. No cracks either in the enamel structure or in the adhesive were observed.

\section{Discussion}

In the first part of the present study shear bond strength of three different light cure bonding adhesives have been evaluated. On completion of bonding, the specimens were stored in distilled water for 24 hours. Rawls [4] has mentioned that even though photopolymerizable composites set by light activation, the maximum bond strength of composite to tooth structure might not occur for at least 24 hours. The same is true for glass ionomer materials because the acid base reaction continues after the initial set. Summers A, et al. [5] found that the shear bond strength of both the composite and resin modified glass ionomer cement were significantly higher at 24 hours than at 30 minutes after bonding.

The present study showed that conventional light cure composite adhesive Transbond-XT had the maximum bond strength of 22.6 MPa with a standard deviation of 3.4 MPa followed by Transbond Plus Self-etching Primer at $19.6 \mathrm{MPa}$ with a standard deviation of $3.42 \mathrm{MPa}$ and
Resin Modified Glass Ionomer Cement at $13.89 \mathrm{MPa}$ with a standard deviation of $2.57 \mathrm{MPa}$.This result is in accordance with the study of Grubisa HSI, et al. [6] In oral cavity bonded brackets are subjected to shear, tensile and torsional forces. Minimum tensile bond strength of 5.9 to 7.9 $\mathrm{MPa}$ would be adequate to resist these treatment forces [7]. According to Lopez JI [8] minimum bond strength of 7Mpa is successful for clinical bonding.

All mean shear bond strength values of the light cure bonding agents used in the present study were above the minimal requirements as mentioned above and hence, are within clinically acceptable range. Profit, Fields and Nixon WL, et al. [9] showed that in balanced faces, posterior teeth were subjected to greater masticatory forces, with forces of around $30 \mathrm{kgf}$ being exerted. In the present study, the mean force in kilogram-force (kgf) at the time of debonding the tubes in Group-A was in the range of $35.3 \mathrm{kgf}-47.7 \mathrm{kgf}$ (Table 2) and in Group-B it was in the range of 30.4kgf-42.8kgf (Table 3) and in Group-C it was 20.8-30.4kgf (Table 4). The results of the present study corroborated with the results of Vilchis RJS, et al. [10] who compared the shear bond strength of conventional acid etching light cure composites (Transbond-XT) with four other self-etching primers. All yielded shear bond strength values higher than the bond strength suggested for routine clinical treatment.

Group-C had least mean shear bond strength of 13.89 MPa with standard deviation of $2.56 \mathrm{MPa}$ (Table 4) but still above the minimal requirements as mentioned by Reynolds [7]. This is in accordance with the study of SE Owens SE, et al. [11] who compared the shear bond strength of two conventional acid etching light cure composites (Transbond-XT) and (Enlight) with resin modified glass ionomer cement, (G.C. Fuji Ortho LC). The most popular bonding systems used in orthodontics are based on micromechanical retention principle which require etching of the enamel surface with phosphoric acid which irreversibly removes several microns of the enamel surface to a non-uniform depth.

Recently, new bonding adhesives self-etching or acidic primers combined with conventional adhesives and conditioning the enamel surface with polyacrylic acid before bonding with resin-modified glass ionomer cements were introduced. These products cause less harm to the enamel surface [12]. The purpose of the second part of the study was to investigate by scanning electron microscopy (SEM) the effect of three light cure adhesives on the surface topography of the enamel. Scanning electron microscope (SEM) used in this study was an Environmental scanning electron microscope 


\section{Open Access Journal of Dental Sciences}

(ESEM) and the advantage of using this is that wet samples in a water vapour environment can be examined without sputter coating of the enamel surfaces $[13,14]$.

Group-A, the SEM images showed an interface between enamel and adhesive with many long, thick resin tags that has penetrated into the enamel surface Figure $15 \mathrm{~b}$ and cracks on the superficial enamel surface (Figures 15(a-c)). Group-B showed resin tags which were thinner, fewer and shorter (Figure 16b). Cracks were observed in the remnant adhesive structure only (Figure 16a). Group$\mathrm{C}$ showed no visible tags (Figure 17b). A distinct border between the enamel and the glass ionomer appeared. No cracks either in the enamel structure or in the remnant adhesive were observed (Figure 17a). Longer and thicker tags were visible with the phosphoric acid treatment compared with the self-etching system, similar to the observations by Torii, et al. [15]

Cracks in the enamel surface was observed only in Group-A and the reason for this can be too high shear bond strength levels that carry the risk for enamel fracture during debonding process. These findings corroborate with the findings of Douglas R, et al. [16] who showed that the greatest frequencies for enamel fracture upon debonding occurred in the groups showing the highest bond strengths i.e. Transbond-XT in their study.

\section{Limitations}

The main limitation of this study is that it is an in-vitro study. However, the findings of this study can be utilised for conducting long term, in-vivo prospective studies with a larger sample size. The other limitation includes the extracted teeth derived from different individuals which may vary in degree of calcification and hence, difference may be observed in the parameters studied. This can be overcome by conducting split mouth studies comparing various bonding materials.

\section{Conclusions}

The inference that can be drawn from the present study is that conventional light cure adhesives have the highest shear bond strength followed by self-etching primer composite adhesives and resin modified glass ionomer cements but all the materials used in the present study had the minimal shear bond strength required for orthodontic bonding. All the bonding systems used in the present study induced different effects on the enamel structure. The possible lower bond strength of the bonding systems based on conditioning the enamel surfaces with a self-etching primer or a polyacrylic acid might be outweighed by fewer irreversible effects on the enamel structure on debonding. The adequate bond strength of glass ionomers, less deleterious effects on the enamel and sustained fluoride release make it a good option for routine use in orthodontics. Further in-vivo evaluation following a full period of routine orthodontic treatment is required to validate the findings of the present study.

\section{Availability of Data and Materials: In-vitro} study

Ethics Approval and Consent to Participate: The study design was approved by the Institutional ethical committee at Armed Forces Medical College, Pune, India. Consent to participate-not applicable as it is an invitro study.

\section{References}

1. Buonocore MG (1955) A simple method of increasing the adhesion of acrylic filling materials to enamel surface. J Dent Res 34(6): 849-853.

2. Buonocore MG, Matsui A, Gwinnett AJ (1968) Penetration of resin dental materials into enamel surfaces with references to bonding. Arch Oral Biol 13(1): 61-70.

3. Newman GV (1965) Epoxy adhesive for orthodontic attachments: A progress Report. Am J Orthod 51(12): 901-912.

4. Anusavice K (2003) Phillips science of dental materials. $11^{\text {th }}($ Edn.), St. Louis: Saunders, pp: 143150.

5. Summers A, Kao E, Gilmore J, Gunel E, Ngan P (2004) Comparison of bond strength between a conventional resin adhesive and a resin modified glass ionomer adhesive: an in-vitro and in-vivo study. Am J Orthod Dentofacial Orthop 126(2): 200-206.

6. Grubisa HSI, Heo G, Raboud D, Glover KE, Major PW (2004) An evaluation and comparison of orthodontic bracket bond strengths achieved with self-etching primer. Am J Orthod Dentofacial Orthop 126(2): 213219.

7. Reynolds IR (1975) A review of direct Orthodontic bonding. Br J Orthod 2(3):171-178. 
8. Lopez JI (1980) Retentive shear strengths of various bonding attachment bases. Am J Orthod 77(6): 669678.

9. Proffit WR, Fields HW, Nixon WL (1983) Occlusal forces in normal and long-face adults. J Dent Res 62(5): 566-570.

10. Vilchis RJS, Yamamto S, Noriyuki K, Yamamoto $\mathrm{K}$ (2009) Shear bond strength of orthodontic brackets bonded with different self-etching adhesives. Am J Orthod Dentofacial Orthop 136(3): 425-430.

11. Owens SE, Miller BH (2000) A comparison of shear bond strengths of three visible light cured orthodontic adhesive. Angle Orthod 70(5): 352-356.

12. Yamada R, Hayakawa T, Kasai K (2002) Effect of using self-etching primer for bonding orthodontic brackets. Angle Orthod 72(6): 558-564.
13. Risnes S (1987) Multiplane sectioning and scanning electron microscopy as a method for studying the three-dimensional structure of mature dental enamel. Scanning Microsc 1(4): 1893-1902.

14. Li C, Risnes S (2004) SEM observations of Retzius lines and prism cross-striations in human dental enamel after different acid etching regimes. Arch Oral Biol 49(1): 45-52.

15. Torii Y, Itou K, Hikasa R, Iwata S, Nishitani Y (2002) Enamel tensile bond strength and morphology of resin-enamel interface created by acid etching system with or without moisture and self-etching priming system. J Oral Rehabil 29(6): 528-533.

16. Douglas R, Timothy FF, Mamandras FA (2001) Comparison of bond strength of three adhesives: composite resin, hybrid GIC, and glass-filled GIC. Am J Orthod Dentofacial Orthop 119(1): 36-42. 